\title{
Advances in Immunotherapy and Molecular Targeted Therapy for Castration Resistant Prostate Cancer
}

\author{
Hai Hao ${ }^{1,2}$, Pan Wenhai ${ }^{1,2, *}$ \\ ${ }^{1}$ Department of Urology, Guang Zhou Red Cross Hospital, Guang Zhou, China \\ ${ }^{2}$ Medical College, Jinan University, Guang Zhou, China \\ Email address: \\ 690474778@qq.com (Hai Hao), 18928900388@189.CN (Pan Wenhai) \\ ${ }^{*}$ Corresponding author
}

To cite this article:

Hai Hao, Pan Wenhai. Advances in Immunotherapy and Molecular Targeted Therapy for Castration Resistant Prostate Cancer. International Journal of Chinese Medicine. Vol. 4, No. 3, 2020, pp. 67-70. doi: 10.11648/j.ijcm.20200403.14

Received: August 31, 2020; Accepted: September 15, 2020; Published: September 23, 2020

\begin{abstract}
Background: Prostate cancer is one of the most common cancer threats to men. The incidence rate and mortality rate are second only to lung cancer in Europe and America. At present, the 5-year survival rate of prostate cancer is only $29 \%$ when metastasis occurs. Prostate cancer seriously endangers the health of male patients. Early prostate cancer surgery and radiotherapy and chemotherapy effect is better, advanced endocrine therapy effect is better, because of drug resistance, eventually progress to castration resistant prostate cancer (CRPC). Although new drugs such as abiraterone acetate, enzalutamide, apalutamide and darutamide have brought new hope to patients with CRPC, when CRPC progresses to metastatic castration resistant prostate cancer (mCRPC), the effect of endocrine therapy is limited. Objective: Immunotherapy, immunosuppressive checkpoint inhibitors and molecular targeted therapy bring new hope to patients with CRPC. This article will review the new progress of immunotherapy and molecular targeted therapy. Methods: A number of clinical and experimental studies were reviewed to indicate the novel advancement in the progressive therapy of CRPC. Results: Immunotherapy, immunosuppressive checkpoint inhibitors and molecular targeted therapy can improve median survival time and have a decrease of PSA level. Conclusion: With the advancements in CRPC therapy made by the researchers, some novel potential methods will occupy more and more important position in the treatment of CRPC.
\end{abstract}

Keywords: Castration Resistant Prostate Cancer, Immunotherapy, Molecular Targeted Therapy

\section{Introduction}

Prostate cancer remains the second most common cause of cancer death in the United States [1]. It is estimated that about 31000 men died this year from metastatic castrated prostate cancer (mCRPC), a deadly form of the disease [1]. Since the approval of radium-223 in 2013, no new treatment has been approved in this field; however, there has been significant progress and expansion in the treatment category for morpc. This review will focus on two new and developing therapeutic areas that have the potential to revolutionize the management of mCRPC: immunotherapy, immunocheckpoint inhibitors, and poly ADP ribose polymerase (PARP) inhibitors targeted therapy. Immunotherapy and the use of PARP inhibitors may change the future prospects and prognosis of $\mathrm{mCRPC}$ patients $[2,3]$.

\section{Immunotherapy}

Prostate cancer is a disease in which cancer vaccines have shown a birth advantage, Sipuleucel-T is a personalized prostate cancer vaccine and exposed to the patient's own antigen presenting cells. It enters into a fusion peptide composed of prostate acid phosphatase (Pap) fused with granulocyte and macrophage colony-stimulating factor. It has been approved for use in asymptomatic and mild patients with $\mathrm{CRPC}$, and plays a therapeutic role by inducing cellular autoimmune regulation. A randomized, multicenter, double-blind, phase III clinical study compared the treatment group with the placebo group. The median survival time of the treatment group was 4.1 months longer than that of the placebo group. The 3-year survival rate of the treatment group was $31.7 \%$, significantly higher than that of the placebo group 
(23\%) [4]. A small sample phase III clinical study with sipuleucel-T suggests a tendency to prolong the survival of patients with CRPC [5]. In April 2010, FDA approved the treatment for patients with mild or asymptomatic mCRPC. Its advantage is that no matter whether the patient has received treatment or not, the drug will produce antigen-specific immune response [6]. However, the complexity of vaccine preparation process and the slightly expensive drug cost restrict its wide clinical application.

Virus based vaccine is an immunotherapy strategy, which uses recombinant virus vector carrying TAA gene sequence to simulate the natural infection of host immune cells, thus causing specific immune response against tumor antigen [7]. Proctvac (TRICOM) is a vaccine based on poxvirus. It consists of recombinant attenuated vaccinia and fowlpox virus enhancer. It encodes TAAs (PSA) and three costimulatory proteins: B7-1 (CD80), lymphocyte function associated antigen 3 (LFA-3) (CD58) and intercellular adhesion molecule-1 (ICAM-1) (CD54)[8]. Gulley et al [9] (results of the recently published trial; patients were randomly divided into three groups: PROSTVAC, PROSTVAC + GM-CSF or placebo. The median overall survival was not affected by either treatment $(34.4,33.2$, and 34.2 months, respectively). The proportion of patients with no event (AWE) at 6 months was similar $(29.4 \%, 28.0 \%$ and $30.3 \%$, respectively). Overall, prostvac is safe, but it does not affect the overall survival of mCRPC patients or the proportion of patients with no events [9].

\section{Immune Checkpoint Inhibitors}

The intensity of immune response mediated by $\mathrm{T}$ cells depends on the balance between CO stimulation and inhibition signals. Immune checkpoint is a kind of molecular substance that prevents $\mathrm{T}$ cells from over activation in the immune system. Malignant tumor cells will overexpress these molecules and further inhibit the killing effect of the immune system. Immune checkpoint inhibitors inhibit tumor cells by inhibiting immune checkpoint molecules and reactivating the killing immunity of $\mathrm{T}$ cells [10]. Pembrolizumab is a monoclonal antibody against PD-1, which can prevent PD-L1 from binding to PD-1 of activated T cells, leading to tumor cell death. Recent studies support the effectiveness of PD-1 inhibitors in some mcrpc patients. It has been reported that $80 \%$ (4 / 5) of merpc patients with MSI-H (highly microsatellite instability) received nivolumab or pembrolizumab, and PSA decreased by more than $50 \%$ [11]. A recent study of CRPC and tumor samples from more than 1000 men found that $2.2 \%$ of the samples had high MSI sensor scores, while the other $9 \%$ had uncertain dmmr scores. Eleven MSI-H / dmmr mCRPC patients received anti-PD-1 / PD-L1 treatment. Six men $(54.5 \%)$ had a decrease of PSA level by more than $50 \%$, and 4 of them had radiation reaction [11]. In the keynote- 028 study, pembrolizumab has also been tested as a monotherapy (without enzalutamide) in patients with advanced PCA. The IB basket trial evaluated the efficacy and safety of pembrolizumab, involving 477 patients with multiple cancers, including mCRPC [12]. The prostate cancer cohort consisted of 23 patients with mCRPC who had to have measurable disease and tissue PD-L1 expression $\geq 1 \%$ (in tumors or immune cells). In these 23 patients, the objective response rate was $13 \%(3 / 23)$, and $39 \%$ of the patients were stable for more than 12 weeks. In patients with objective remission, the median duration of remission was 59 weeks (range, 28-62 weeks). The median OS was 8 months, which was a reasonable OS considering that $74 \%$ of patients had received two or more systemic treatments for metastatic CRPC disease [12].

On May 23, 2017, the US FDA announced that it would accelerate the approval of keytruda, an antibody against PD-1 [13]. It is the first time that FDA does not use tumor site as reference and only relies on biomarkers for the treatment of advanced or metastatic solid tumors in adults and children with high microsatellite instability or mismatch repair gene defects, and the effective rate is as high as $40 \%$. Two of the most promising strategies for combined immunotherapy of mcrpc are to combine two different checkpoint inhibitors, or to combine one checkpoint inhibitor with a novel antiandrogen, enzalutamide, which has been approved for use in morpc. Checkmate-650 studied the use of a PD-1 inhibitor nivolumab in combination with anti CTLA4 antibody ipilimumab for the second or third line treatment of mCRPC [14]. In this study, men who had previously received chemotherapy had an objective response rate of $10 \%$ (three out of 30 ), but it was more promising that $26 \%$ (6 out of 23 ) of men at the beginning of chemotherapy had an objective response rate [14]. In the keynote-365 study, pembrolizumab combined with enzalutamide was used in patients with mCRPC resistant to abiraterone (before chemotherapy). The study included 69 patients. The PSA response rate was 33\% (18 of 54 evaluable patients) and the objective response rate was $20 \%$ ( 5 of 25 evaluable patients) [15].

\section{Molecular Targeted Therapy}

In recent years, molecular targeted therapy has become one of the new research hotspots in tumor therapy. It mainly plays a therapeutic role by selectively inhibiting tumor specific molecular targets. Molecular targeted drugs for the treatment of CRPC are gradually used in clinical practice. The inhibition of poly ADP ribose polymerase (PARP) leads to the accumulation of DNA single strand breaks, and eventually leads to the damage of replication fork. Both PARP1 and PARP2 mediate the repair of single strand breaks through base excision repair. PARP inhibitors lead to cancer cell death through "synthetic lethality", that is, the combination of PARP inhibition and homologous recombination gene mutation in tumor may lead to the loss of compensatory DNA repair mechanism, leading to cancer cell death due to irreparable DNA damage [16]. In normal cells, the cleavage was repaired by error free homologous recombination. When BRCA fails in homologous recombination pathway, PARP inhibitors induce synthesis death by blocking base excision repair [17-19]. PARP inhibitors have been approved for BRCA1 and BRCA2 (BRCA) mutations in breast and ovarian cancer [20, 21]. 
Evidence of activity is also shown in patients with metastatic castration resistant prostate cancer (mCRPC) with BRCA mutations, based on a good combined lethal effect [3].

In preclinical studies, PARP inhibitors have been shown to inhibit tumor growth and can be used in hormone insensitive prostate cancer patients and prostate cancer patients with distant metastasis. In a toparpii phase I trial, a total of 50 patients with mCRPC were enrolled; all patients had been treated with docetaxel, $49(98 \%)$ were treated with abitiron or enzalutamide, and 29 (58\%) were treated with carbamazepine. The primary endpoint was response rate, or a reduction in PSA levels by at least $50 \%$, or a reduction in circulating tumor cell count from 5 or more cells per $7.5 \mathrm{ml}$ of blood to less than 5 cells per $7.5 \mathrm{ml}$ of blood. Overall, results of the 49 evaluable patients, $16(33 \%$; 95\% CI 20-48) responded, of which 12 received study treatment for more than 6 months. Next generation sequencing found homozygous deletions, harmful mutations, or both of the DNA repair genes (including BRCA1 / 2, ATM, Fanconi anemia gene, and CHEK2) in 16 (33\%) of 49 evaluable patients. Of the 16 patients, $14(88 \%)$ responded to olaparib, including all 7 patients with BRCA2 deletion (4 with biallelic loss and 3 with germline mutation) and 4 / 5 of ATM abnormalities. The specificity was $94 \%$. This suggests that olaparib, a PARP inhibitor, can lead to a high response rate in patients with prostate cancer who are no longer responsive to standard treatment and have DNA repair gene defects [22]. Recently, a randomized, placebo-controlled phase II clinical trial compared the use of abiolone alone with that of abitelone combined with olaparib in 142 male patients with mcrpc. It showed a tendency to prefer the combination of abiraterone and olaparib, and there was no correlation between the homologous recombination and the treatment group [23]. Therefore, the general concept is to use adjuvant therapy to reduce immunosuppressive induced drug resistance or insensitivity. In a multicenter phase II study [24] of 298 solid tumor patients treated with olaparib, 8 patients with metastatic CRPC had BRCA1 / 2 mutation, and the orr was as high as $50 \%$. It has been found that patients with metastatic CRPC with homologous recombination repair (HRR) mutation can benefit significantly from the targeted therapy of olaparib [25].

Due to the efficacy of olaparib in the treatment of advanced prostate cancer, a large number of researchers have devoted themselves to the combination of PARP inhibitors and other methods. Preclinical data suggest that PARP inhibitors and androgen receptor antagonists have potential synergistic effects regardless of the HRR status of prostate cancer [26]. In a randomized, placebo-controlled phase II clinical study, the efficacy of olaparib combined with abiraterone was evaluated [27]. It has been reported that olaparib combined with abiraterone is beneficial for longer radioprogression free survival (RPFS) (13.8 vs 8.2 months). The recently updated keynote-365 cohort (nct02861573) reported that in M1 CRPC patients after docetaxel treatment, the disease control rate (DCR) of olaparib combined with pembrolizumab for more than 6 months was 32\% [28]. Rae et al [29] found that the combination of rucaparib, olaparib and HSP90 inhibitors
(17-DMAG) could inhibit the sensitivity of prostate cancer cells. Zhang et al [30] also found that platinum combined with olaparib may be a new treatment option for prostate cancer patients with MYCN and aurka gene mutations. Based on the above data, more and more clinical trials have been carried out to further confirm the efficacy of olaparib monotherapy or combination therapy for CRPC.

PARP inhibitors initially work well, but they also develop resistance. One possible mechanism is acquired BRCA2 reverse mutation, in which the former BRCA-2 deficient tumor cells can reach BRCA2 level due to the constant selective pressure of PARP inhibition [31]. Serial sequencing of circulating cell-free (CF) DNA can help to monitor these reversals in a noninvasive manner. In addition to this mechanism, the loss of MLL3 / 4 complex protein and PTIP helps to stabilize the replication fork and protect DNA from degradation [32].

\section{Conclusion}

CRPC is the final outcome of most prostate cancer patients. Molecular targeted drugs, Immune checkpoint inhibitors and immunotherapy have been used in clinical practice. The treatment of CRPC will certainly bring more hope for survival for more and more patients. In the specific treatment, we should consider the survival expectation, general condition, economic ability and other factors of patients, and carry out individualized treatment.

\section{References}

[1] Siegel RL, Miller KD, Jemal A. Cancer statistics, 2019. Ca A Cancer Journal for Clinicians. 2019.

[2] Topalian S, Drake C, Pardoll D. Immune checkpoint blockade: a common denominator approach to cancer therapy. Cancer cell. 2015; 27 (4): 450-61. doi: 10.1016/j.ccell.2015.03.001.

[3] Lord C, Ashworth A. PARP inhibitors: Synthetic lethality in the clinic. Science (New York, NY). 2017; 355 (6330): 1152-8. doi: 10.1126/science.aam7344.

[4] PW K, CS H, ND S, ER B, EJ S, DF P, et al. Sipuleucel-T immunotherapy for castration-resistant prostate cancer. The New England journal of medicine. 2010; 363 (5): 411-22. doi: 10.1056/NEJMoa1001294.

[5] Higano CS, Schellhammer PF, Small EJ, Burch PA, Nemunaitis J, Yuh L, et al. Integrated data from 2 randomized, double-blind, placebo-controlled, phase 3 trials of active cellular immunotherapy with sipuleucel-T in advanced prostate cancer. Cancer. 2009; 115 (16): 3670-9. doi: $10.1002 /$ cncr.24429.

[6] Simondsen K, Kolesar J. New treatment options for castration-resistant prostate cancer. Am J Health Syst Pharm. 2013; 70 (10): 856-65. doi: 10.2146/ajhp110586.

[7] Sultan H, Fesenkova VI, Addis D, Fan AE, Kumai T, Wu J, et al. Designing therapeutic cancer vaccines by mimicking viral infections. Cancer immunology, immunotherapy: CII. 2017; 66 (2): 203-13. doi: 10.1007/s00262-016-1834-5. 
[8] Madan RA, Bilusic M, Heery C, Schlom J, Gulley JL. Clinical evaluation of TRICOM vector therapeutic cancer vaccines. Seminars in oncology. 2012; 39 (3): 296-304. doi: 10.1053/j.seminoncol.2012.02.010.

[9] Gulley JL, Borre M, Vogelzang NJ, Ng S, Agarwal N, Parker $\mathrm{CC}$, et al. Phase III Trial of PROSTVAC in Asymptomatic or Minimally Symptomatic Metastatic Castration-Resistant Prostate Cancer. J Clin Oncol. 2019; 37 (13): 1051-61. doi: 10.1200/jco.18.02031.

[10] Xu Biaobo, He yijing, Wang Weili, Zhou Chengfang, Xie Shangchen, Shen Dongya, et al. Advances in clinical treatment of tumor immune checkpoint inhibitors. Chinese clinical pharmacology and therapeutics. 2016; 21 (02): 218-24.

[11] Smits M, Doelen MJvd, Westdorp H, Oort IMv, Sedelaar M, Heijden AVD, et al. Immunological and genomic correlates of response to anti-PD1 checkpoint therapy in mismatch proficient and deficient patients with metastasized castration resistant prostate cancer. journal of clinical oncology. 2018; 36: 248-. doi: 10.1200/JCO.2018.36.6_SUPPL.248.

[12] Hansen AR, Massard C, Ott PA, Haas NB, Lopez J, Ejadi S, et al. Pembrolizumab for patients with advanced prostate adenocarcinoma: Preliminary results from the KEYNOTE-028 study. Annals of Oncology. 2016; 27.

[13] Pai-Scherf L, Blumenthal G, Li H, Subramaniam S, Mishra-Kalyani P, He K, et al. FDA Approval Summary: Pembrolizumab for Treatment of Metastatic Non-Small Cell Lung Cancer: First-Line Therapy and Beyond. The oncologist. 2017; 22 (11): 1392-9. doi: 10.1634/theoncologist.2017-0078.

[14] Sharma P, Pachynski RK, Narayan V, Flechon A, Gravis G, Galsky MD, et al. Initial results from a phase II study of nivolumab (NIVO) plus ipilimumab (IPI) for the treatment of metastatic castration-resistant prostate cancer (mCRPC; CheckMate 650). journal of clinical oncology. 2019; 37: 142-. doi: 10.1200/JCO.2019.37.7 SUPPL.142.

[15] Fong PCC, Retz M, Drakaki A, Massard C, Berry WR, Romano E, et al. Pembrolizumab (pembro) plus enzalutamide (enza) in abiraterone (abi)-pretreated patients (pts) with metastatic castrate resistant prostate cancer (mCRPC): Cohort $\mathrm{C}$ of the phase 1b/2 KEYNOTE-365 study. journal of clinical oncology. 2019; 37: 5010-. 10.1200/JCO.2019.37.15_SUPPL.5010.

[16] Ashworth A, Lord C. Synthetic lethal therapies for cancer: what's next after PARP inhibitors? Nature reviews Clinical oncology. 2018; 15 (9): 564-76. doi: 10.1038/s41571-018-0055-6.

[17] Javle M, Curtin NJ. The potential for poly (ADP-ribose) polymerase inhibitors in cancer therapy. Therapeutic advances in medical oncology. 2011; 3 (6): 257-67. doi: $10.1177 / 1758834011417039$.

[18] Helleday T. The underlying mechanism for the PARP and BRCA synthetic lethality: clearing up the misunderstandings. Mol Oncol. 2011; 5 (4): 387-93. doi: 10.1016/j.molonc.2011.07.001.

[19] CJ L, A A. PARP inhibitors: Synthetic lethality in the clinic. Science (New York, NY). 2017; 355 (6330): 1152-8. doi: 10.1126/science.aam7344

[20] SA C, AV T. PARP Inhibitors and the Evolving Landscape of Ovarian Cancer Management: A Review. BioDrugs: clinical immunotherapeutics, biopharmaceuticals and gene therapy.
2019; 33 (3): 255-73. doi: 10.1007/s40259-019-00347-4.

[21] MYT K, Y W, JV V. PARP Inhibitors as a Therapeutic Agent for Homologous Recombination Deficiency in Breast Cancers. Journal of clinical medicine. 2019; 8 (4). doi: $10.3390 / \mathrm{jcm} 8040435$.

[22] Mateo J, Carreira S, Sandhu S, Miranda S, Mossop H, Perez-Lopez R, et al. DNA-Repair Defects and Olaparib in Metastatic Prostate Cancer. N Engl J Med. 2015; 373 (18): 1697-708. doi: 10.1056/NEJMoa1506859.

[23] ES A. Abiraterone plus olaparib in prostate cancer: a new form of synthetic lethality? The Lancet Oncology. 2018; 19 (7): 860-1. doi: 10.1016/s1470-2045(18)30409-1.

[24] Sunkel B, Wu D, Chen Z, Wang CM, Liu X, Ye Z, et al. Integrative analysis identifies targetable CREB1/FoxA1 transcriptional co-regulation as a predictor of prostate cancer recurrence. Nucleic Acids Res. 2017; 45 (11): 6993. doi: $10.1093 / \mathrm{nar} / \mathrm{gkx} 282$.

[25] De Bono JS, Hussain M, Thieryvuillemin A, Mateo J, Sartor AO, Chi KN, et al. PROfound: A randomized Phase III trial evaluating olaparib in patients with metastatic castration-resistant prostate cancer and a deleterious homologous recombination DNA repair aberration. Journal of Clinical Oncology. 2017; 35.

[26] M A, F T, HI Z, K S, E G, CE M, et al. Synthetic lethality between androgen receptor signalling and the PARP pathway in prostate cancer. Nature communications. 2017; 8 (1): 374. doi: 10.1038/s41467-017-00393-y.

[27] Clarke N, Wiechno P, Alekseev B, Sala N, Jones R, Kocak I, et al. Olaparib combined with abiraterone in patients with metastatic castration-resistant prostate cancer: a randomised, double-blind, placebo-controlled, phase 2 trial. The Lancet Oncology. 2018; 19 (7): 975-86. doi: $10.1016 / \mathrm{s} 1470-2045(18) 30365-6$

[28] Yu EY, Massard C, Retz M, Tafreshi A, Galceran JC, Hammerer P, et al. Keynote-365 cohort a: Pembrolizumab (pembro) plus olaparib in docetaxel-pretreated patients (pts) with metastatic castrate-resistant prostate cancer (mCRPC). Journal of Clinical Oncology. 2019; 37 (7_suppl): 145-. doi: 10.1200/JCO.2019.37.7_suppl.145.

[29] C R, RJ M. Evaluation of the radiosensitizing potency of chemotherapeutic agents in prostate cancer cells. International journal of radiation biology. 2017; 93 (2): 194-203. doi: 10.1080/09553002.2017.1231946.

[30] W Z, B L, W W, L L, BM B, SP B, et al. Targeting the MYCN-PARP-DNA Damage Response Pathway in Neuroendocrine Prostate Cancer. Clinical cancer research: an official journal of the American Association for Cancer $\begin{array}{lllll}\text { Research. 2018; } 24 & \text { (3): 696-707. doi: }\end{array}$ 10.1158/1078-0432.ccr-17-1872.

[31] D Q, JJ A, AW W, V K, A F, P L, et al. BRCA2 Analysis of Circulating Cell-Free DNA Identifies Multiclonal Heterogeneity of Reversion Mutations Associated with Resistance to PARP Inhibitors. Cancer discovery. 2017; 7 (9): 999-1005. doi: 10.1158/2159-8290.cd-17-0146.

[32] A RC, E C, X D, E G, AA D, JE L, et al. Replication fork stability confers chemoresistance in BRCA-deficient cells. Nature. 2016; 535 (7612): 382-7. doi: 10.1038/nature18325. 